\title{
Head-Out Spirometry Accurately Monitors the Course of Pseudomonas aeruginosa Lung Infection in Mice
}

\author{
Florian Wölbeling Antje Munder Frauke Stanke Burkhard Tümmler \\ Ulrich Baumann \\ Pediatric Pulmonology and Neonatology, Hannover Medical School, Hannover, Germany
}

Key Words

C57BL6/J mice $\cdot$ Pseudomonas aeruginosa $\cdot$ Head-out spirometry

\begin{abstract}
Background: Classic infection models in rodents use lethal doses of bacteria as inocula, thus creating models which are rarely comparable to the clinical situation. Moreover, single time-point evaluation requires killing of the animals, necessitating large numbers of animals. Longitudinal parameters such as temperature appear to have a relatively low accuracy. Spirometry might be an accurate method to assess the course of a bacterial lung infection without the necessity to sacrifice the animals. Objectives: We measured lung function in C57BL/6JZtm mice following intratracheal infection with Pseudomonas aeruginosa and compared it to physiological parameters and lung histology. Methods: Head-out spirometry measuring 14 parameters was performed on C57BL6/J mice for eight days following a $P$. aeruginosa lung infection. Additionally rectal temperature, body weight and condition were assessed together with histological data and bacteriological clearance. Results: Several spirometric parameters were significantly altered for more than $72 \mathrm{~h}$ after inoculation, which was four times longer than observed alterations in physiological parameters such as temperature. Volume (amount of air inspired) decreased more than seven-
\end{abstract}

\section{KARGER}

Fax +4161306 1234 E-Mail karger@karger.ch www.karger.com
(C) 2010 S. Karger AG, Basel

$0025-7931 / 10 / 0804-0340 \$ 26.00 / 0$

Accessible online at:

www.karger.com/res fold within $6 \mathrm{~h}$ after inoculation and required $72 \mathrm{~h}$ to recover, rendering it the most sensitive spirometric parameter investigated. Spirometric and histological data correlated well. Conclusions: Our findings suggest that non-invasive headout spirometry is a reliable and highly sensitive method to longitudinally assess the course of bacterial lung infections.

Copyright $\odot 2010$ S. Karger AG, Basel

\section{Introduction}

Spirometry is an established and extensively used method to assess pulmonary status in humans with lung pathologies like cystic fibrosis, asthma or COPD [1-4]. In cystic fibrosis and COPD, it is a valuable tool for the diagnosis of an acute exacerbation [5-7]. An imminent threat for patients with cystic fibrosis and COPD is the acquisition of opportunistic pathogens like Pseudomonas aeruginosa $[8,9]$. Prevention or treatment of these infections is a major task. Animal models are frequently utilized in research for a better understanding of the disease and to assist in testing new therapies. However, for ethical and economical reasons care should be taken to reduce or refine the number of needful animal experiments.

Traditionally, longitudinal investigation of bacterial infections in animal models involves the requirement to sacrifice large cohorts of animals to receive information

E-Mail woelbeling.florian@mh-hannover.de 
of the processes happening at various time points [10]. Spirometry visualizes the respiratory health status of the animal without the necessity to sacrifice or anesthetize, thus enabling repeated measurements on the same animal in one experiment.

Several methods of spirometry for rodents exist; each is believed to have its own strengths and weaknesses correlating with their level of invasiveness [11].

Invasive measurements [12] requiring the anesthetization of the mice and the insertion of a probe in the trachea, are known for their superior accuracy at the cost of a high level of stress/damage to the subject and an elaborate technique which limits the use of this method for time course experiments as in bacterial infections. Only a limited number of mice can usually be measured in parallel, thus any putative inter-individual variance may not be easily assessed.

Non-invasive measurements like whole-body plethysmography [13] do not require anesthesia and consequently put the animal under less stress. In this method, mice move freely in a chamber where sensors obtain the respiratory data. Larger cohorts of mice can be measured, but with poorer data quality.

We decided to use another non-invasive method, termed 'head-out spirometry', for our experiments which combines greater accuracy than whole-body plethysmography with the possibility of measuring more mice in parallel than in invasive spirometry at comparable accuracy [14]. In head-out spirometry [15], mice are not anesthetized but are restrained in inserts where only their heads protrude through a pair of membranes. This enables repeated measurements on the same animals within a very short period of time. However, to the best of our knowledge, this method has never been used to monitor the course of bacterial airway infections in mice.

In this paper, we discuss how accurately the method of head-out spirometry monitors the course of acute $P$. aeruginosa airway infection in mice.

\section{Materials and Methods}

\section{Animals}

C57BL/6JZtm mice (Central Animal Laboratory, Hannover Medical School, Germany) were maintained in microisolator cages with filter top lids at $21 \pm 2{ }^{\circ} \mathrm{C}, 50 \pm 5 \%$ humidity and a 14 /10-hour light-dark cycle. They were supplied with autoclaved, acidulated water and fed ad libitum with autoclaved standard diet. All animal procedures were approved by the local animal welfare committee and carried out according to the guidelines of the German regulations for animal protection.
Bacterial Infection

P. aeruginosa strain TBCF10839 [16] was grown in Luria broth at $37^{\circ} \mathrm{C}$ overnight. The optical density of the bacterial suspension was determined and the intended number of colony forming units (CFU) was extrapolated from a standard growth curve. Inocula of $6 \times 10^{5} \mathrm{CFU} / 30 \mu \mathrm{l}$ were prepared by dilution with sterile PBS. Anesthetized mice were inoculated via view-controlled intratracheal instillation [17].

\section{Measured Parameters}

Head-out spirometry with 14 parameters was performed on conscious restrained mice. Additionally, body weight and rectal temperature of the mice were measured at each time point. Behavior and general condition were scored as described previously in the study of Munder et al. [18]. More detail on these methods, including a video, is provided in the supplementary material to this paper, which is available online at www. karger.com/doi/10.1159/000319442.

\section{Histology, CFU Organ Count}

Three mice not belonging to the spirometry group were sacrificed and investigated at each of the timepoints 4, 8, 12, 24 and $48 \mathrm{~h}$ after inoculation. The right lung was fixed and stained for histological analysis; the left lung was homogenized in sterile PBS and plated on Luria broth agar plates for CFU determination. Incubation was performed overnight at $37^{\circ} \mathrm{C}$. Homogenization and plating of the liver, brain and spleen was performed in a similar manner.

\section{Spirometry Hardware}

A custom-made glass corpus with four inserts enabling the parallel investigation of four mice was used. We attached to one end of the insert two $6 \times 6$ inch $(15.2 \times 15.2 \mathrm{~cm})$ membranes, a Roeko Dental Dam green (silicone, Roeko, Langenau, Germany) and a Hygenic Dental Dam blue (latex, Hygenic, Akron, Ohio, USA), which had holes of a specific diameter corresponding to the neck size of the. The entrance end of the chamber was secured with a screwable cap with a movable glass piston.

For each insert, a pneumotachograph (PTM Type 378/0.9) and a pressure transducer (DLP10 Type 380) were positioned directly above the insert. An amplifier (Transducer Amplifier Module Type 705/1), and an analog-to-digital converter (Data Translation 16 Channels, USB BNC Box, DT9800 BMC Box 16SE) were employed for digitalization of the airflow. All items except the glassware were supplied by Hugo Sachs Elektronik - Harvard Apparatus (March-Hugstetten, Germany). For data acquisition and analysis Notocord Hem (version 4.2.0.241, Notocord Systems, Croissy-sur-Seine, France) was used.

\section{Statistical Analysis}

Since the investigated spirometric parameters did not follow a normal distribution, $p$ values (significant at $p<0.05$ ) with subsequent Bonferroni correction [19] were calculated by 2-sided Monte Carlo simulations (100,000 simulations), following non-parametric tests for connected (Wilcoxon [20]) and non-connected samples (Mann-Whitney U [21]) using SPSS 16 (version 16.0.2, SPSS Inc, Chicago, Ill., USA). 
Table 1. Selected spirometric values and physiological data for the investigated mice prior to infection

\begin{tabular}{lccc}
\hline Parameter, unit & \multicolumn{2}{l}{ Median and interquartile range } & \multirow{2}{*}{$\mathrm{p}^{2}$} \\
\cline { 2 - 3 } & male $(\mathrm{n}=10)$ & female $(\mathrm{n}=13)$ & \\
\hline Tidal volume, $\mathrm{ml}$ & $0.265(0.240-0.281)$ & $0.225(0.210-0.252)$ & $\mathbf{0 . 0 1 8}$ \\
Expiratory time, $\mathrm{ms}$ & $128.92(124.28-132.80)$ & $119.91(111.39-127.11)$ & 0.227 \\
EF50 (flow at 0.5 VTE), $\mathrm{ml} / \mathrm{s}$ & $2.936(2.633-3.118)$ & $2.861(2.620-3.028)$ & 0.086 \\
Respiratory rate, bpm & $262(256-272)$ & $278(260-298)$ & 0.407 \\
Body weight, $\mathrm{g}$ & $32.3(30.8-33.3)$ & $23.1(22.5-24.7)$ & $\mathbf{0 . 0 0 0}$ \\
\hline
\end{tabular}

${ }^{1}$ Data calculated from three independent measurements prior to infection.

${ }^{2}$ Two-sided p values were calculated utilizing Mann-Whitney U test. Significant values $(\mathrm{p}<0.05)$ are marked in bold. For a complete table of all investigated parameters, please refer to the supplementary online materials.

\section{Results}

\section{Bacterial Infection Model}

A total of 23 mice ( 13 female and 10 male) with a mean age of $128 \pm 24$ days was chosen to test for putative gender-associated differences in lung infection. Infection with $6 \times 10^{5} \mathrm{CFU}$ of strain TBCF10839, which is one order of magnitude below the LD50 for this mouse strain and $P$. aeruginosa strain, were sufficient to induce a noticeable infection, while at the same time limiting the number of dead animals. Following infection, rectal temperature dropped to approximately $24^{\circ} \mathrm{C}$ for both genders. Female and male mice reached their minimum temperature at 6 and $10 \mathrm{~h}$ after inoculation, respectively. Recovery to normal values was achieved for females at $18 \mathrm{~h}$ after inoculation and for males at $24 \mathrm{~h}$ after inoculation. Male control mice $(n=14)$ of comparable age $(93 \pm 38$ days), which received the same treatment but an instillation of $\mathrm{NaCl}$ at physiological concentrations (0.9\%), experienced only minor changes in body temperature. The behavioral score reached the maximal value of disturbance at 6 to $8 \mathrm{~h}$ after inoculation for infected females and at 8 to $10 \mathrm{~h}$ after inoculation for males. Recovery was achieved within $24 \mathrm{~h}$ for males and $48 \mathrm{~h}$ for females. Mice in the control cohort showed only minor signs of disturbance at $4 \mathrm{~h}$ after procedure, and were still classified as 'unaffected'.

\section{Spirometric Values of Uninfected Mice}

Table 1 summarizes values for selected spirometric parameters as well as body weight data for the investigated mice taken from three independent measurements prior to infection (median). A complete table of standard values for all 14 investigated spirometry parameters, as well as a more detailed description of the spirometric process, including a video file, is provided in the supplementary online material files.

Although male and female mice differed significantly in weight, only parameters directly reflecting the lung size of the animal like tidal volume (total volume inspired and expired during one breath) and volume (inspired air volume; integral of tidal volume) differed at the 0.05 significance level. However, these differences vanished after correction for multiple testing. All other spirometric parameters did not significantly differ between males and females prior to infection in this study cohort. Females had a slightly faster respiratory rate, which is consistent with their smaller body size. Accordingly, the airflows during inspiration and expiration were smaller as well.

\section{Bacterial Infection and Spirometry}

Following a bacterial lung infection with $6.0 \times 10^{5}$ CFU of $P$. aeruginosa, all parameters showed pathological values with a maximum around 6 to $8 \mathrm{~h}$ after inoculation and a recovery period as long as $72 \mathrm{~h}$ (fig. 1). In the control cohort only minor changes in lung function could be observed, which were already completely reversed $8 \mathrm{~h}$ after the procedure.

To better characterize the time course of lung function values, we determined the maximal deviation from normal (dashed line in the figure) together with a fold-change value (max./min. divided by initial value) and the return to normal values (solid line, Wilcoxon, MC) for males and females.

Consistent with body temperature and behavioral score, females were more rapidly affected. In fact, in most cases the maximal deterioration from normal could already be observed at $6 \mathrm{~h}$ after inoculation, while males 


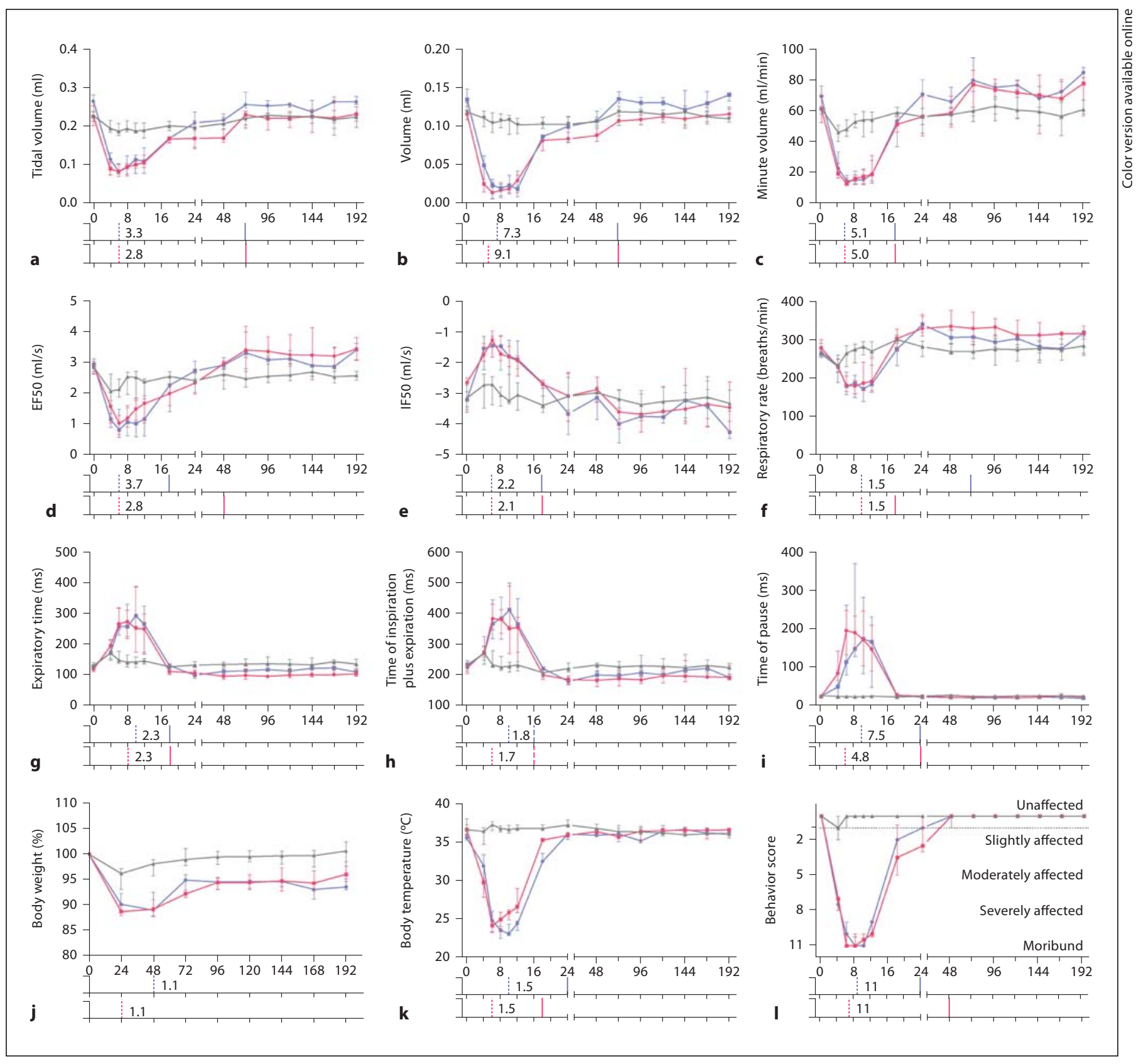

Fig. 1. Median and interquartile range curves for selected spirometric and physiological parameters. Depicted are volume parameters $(\mathbf{a}-\mathbf{c})$, flow parameters $(\mathbf{d}, \mathbf{e})$, respiratory rate $(\mathbf{f})$, time parameters $(\mathbf{g}-\mathbf{i})$, and physiological parameters $(\mathbf{j}-\mathbf{I})$. Abscissas below each graph depict time after inoculation in hours as well as the graphical maximal deviation (dashed line) together with foldchange and return to normal values (solid line) for infected males (blue line; upper axis, $\mathrm{n}=10$ ) and females (red line; lower axis, $\mathrm{n}=13$ ). No statistics were performed for control cohort mice (grey line). Time of inspiration plus expiration (h) did not return to normal values; therefore, a visual return to normal values was depicted by a dashed line. Please refer to the online supplementary material (www.karger.com/doi/10.1159/000319442) for further respiratory curves. 


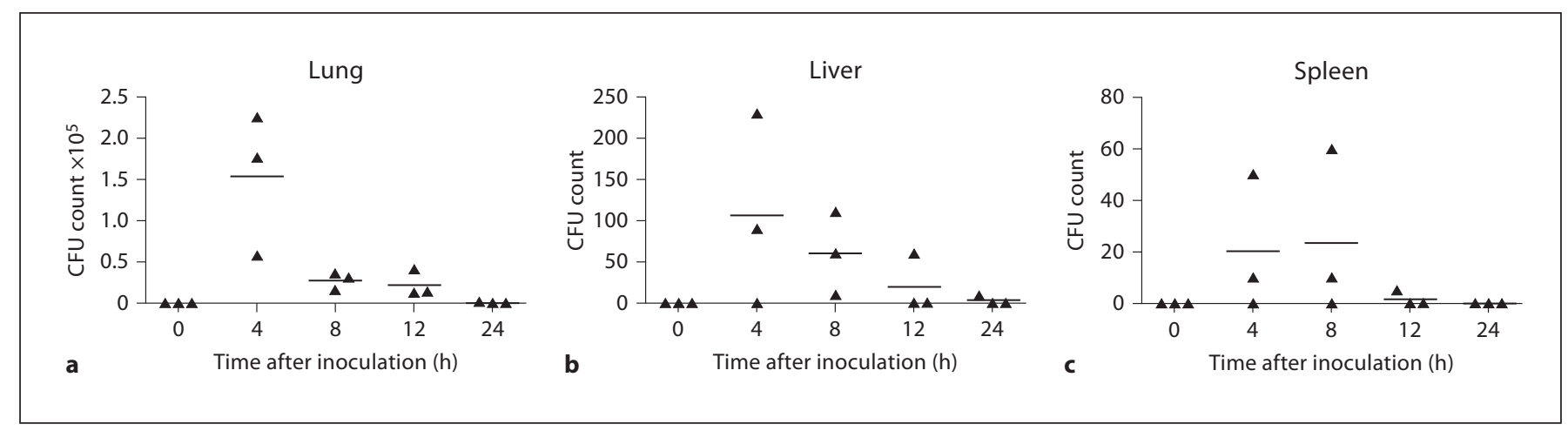

Fig. 2. CFU organ counts in selected organs (mean). Three mice per time point were sacrificed and half of their lungs were homogenized and plated. Since CFUs were only determined in half of the lung, the number of CFU in the graph was doubled. The overall kinetics are quite similar for lung (a) and liver (b). The maximum
CFU count can be observed at $4 \mathrm{~h}$ after infection, with an initial strong decrease until $24 \mathrm{~h}$ after infection. The homogenate of the spleen (c) showed only small amounts of bacteria. The bloodbrain barrier was apparently not penetrated as virtually no bacteria were detected in the brains of the mice (data not shown). were delayed by 2 h. Nonetheless, the overall magnitude of fold changes was almost identical for males and females and not significantly different $(\mathrm{p}=0.8)$ for both genders. The return to values prior to infection was similar for males and females in most parameters, as determined by Wilcoxon tests. The minimum time for a return to normal values was $18 \mathrm{~h}$, with at least five parameters, particularly volume parameters requiring no less than $24 \mathrm{~h}$ for a return to normal values.

An ideal spirometric parameter for monitoring an infection would not only have a significant fold change but also a measurable deviation for a long time. A very robust and possibly the most useful investigated parameter was therefore volume. Graphically judged from the median curves, the maximal deviation for females was at $6 \mathrm{~h}$ after inoculation, and for males it was at $8 \mathrm{~h}$ after inoculation. At that point volume decreased by $89.0 \%$ for females and $86.3 \%$ for males, resulting in a 9.1-fold change for females and a 7.3 -fold change for males from the starting value. A return to normal could be observed at $72 \mathrm{~h}$ after inoculation for both genders.

In summary, most spirometric parameters supported the trend that females were affected earlier but not more strongly in terms of fold change. Return to normal was comparable for both genders. Several spirometric parameters were able to depict infection for considerably longer than physiological parameters such as temperature.

CFU Count

CFU counts in the lungs of mice at specific time points $(0,4,8,12,24 \mathrm{~h})$ after inoculation revealed a maximal bacterial burden in the lungs at $4 \mathrm{~h}$ after inoculation, with about one third $\left(1.8 \times 10^{5} \mathrm{CFU}\right)$ of the inoculated dosage being recovered (fig. 2). Four hours later only a fraction of the amount of bacteria as before was recovered, with a further decrease until at $24 \mathrm{~h}$ after inoculation when almost all bacteria were cleared. For the liver a similar kinetic was observed with markedly smaller CFU, indicating a minimal dissemination into the bloodstream. In the spleen only few bacteria could be recovered. These numbers of bacteria did not correlate with the maximum deviation from normal in the spirometric parameters, which took place between 6 and $10 \mathrm{~h}$ after inoculation.

\section{Histological Analysis}

Paraffin-embedded lung histological slices of the right lungs were assessed regarding the inflammation in the lung parenchyma and in the bronchial areas using a semiquantitative score system (0-5 not visible, 5-20 slight, $20+$ moderate signs of inflammation; fig. 3). In the parenchymal areas of the lung tissue, slight inflammation could be seen at $4 \mathrm{~h}$ after inoculation. The inflammatory peak was observed at $8 \mathrm{~h}$ after inoculation, when nearly the complete lungs showed a moderate purulent alveolar pneumonia, followed by subsequent decrease of inflammation (histological sample slides are provided in the additional information on the model in the supplementary online materials).

For the bronchi, only slight signs of inflammation could be observed, mostly seen as peribronchiolar and only very rarely intrabronchiolar infiltrates, which increased from 4 to $8 \mathrm{~h}$. But unlike in the parenchyma the 


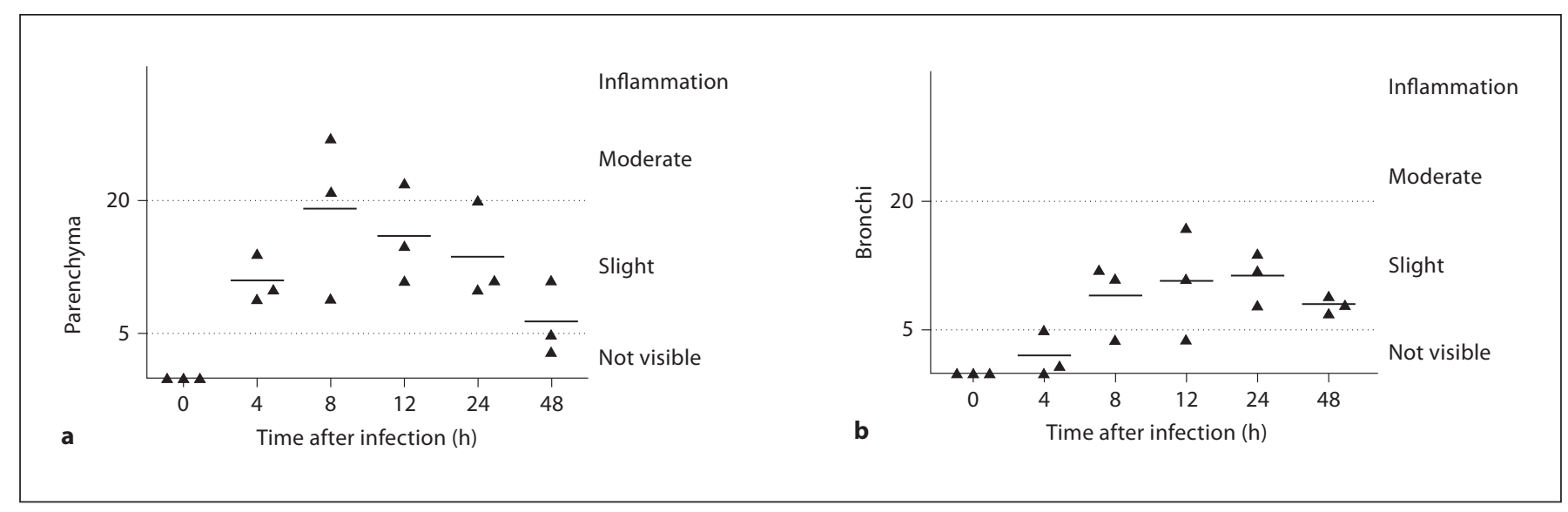

Fig. 3. Morphological analysis of the lung histology samples utilizing a semiquantitive score $(0-5$ not visible, 5-20 slight, $20+$ moderate signs of inflammation). For the parenchyma (a), the maximum signs of inflammation characterized by immune cell infiltrates could be observed at $8 \mathrm{~h}$ after infection with subsequent decrease until $48 \mathrm{~h}$. For the bronchi (b), a slightly different kinetic could be observed with a maximum at $12 \mathrm{~h}$ after infection followed by a decrease in the amount of inflammation. bronchial inflammation still continued to increase until $24 \mathrm{~h}$ after inoculation. Finally, starting at $48 \mathrm{~h}$ after inoculation, a decrease of inflammation could be observed.

In summary, the visual examination of the histological samples resulted in a pattern different from the bacterial burden, whose maximum was observed $4 \mathrm{~h}$ after inoculation. The observed pattern for the parenchyma showed strong similarity to the kinetics of the spirometric data.

\section{Discussion}

In the present study, we demonstrate the utilization of spirometry as a method to monitor bacterial lung infections in rodents. Major findings include that any putative gender differences in the investigated study group were mainly attributed to different initial body weights and consequently different infectious doses per gram mouse. Nevertheless, lung infection with $P$. aeruginosa resulted in a comparable severity in infection, with the only difference being that female mouse alterations peaked about $2 \mathrm{~h}$ earlier. For both genders recovery took as long as $72 \mathrm{~h}$ for selected lung function parameters. Additionally, a correlation between the histological data with the spirometric data could be observed rather than with the CFU count, indicating that the actions of the immune system rather than the bacterial burden are responsible for the deterioration of the lung function.
Compared to more classic outcome parameters, spirometry has some major advantages. As spirometry can be utilized repeatedly on the same animal for continuous monitoring even in the interval of hours, no classic endpoints like LD50 values or bronchoalveolar lavage are needed, effectively reducing lab animal consumption. The infectious dose can also be employed more flexibly even making low-dose experiments possible that more closely mimic the clinical situation of acquired infections. In fact, different infectious doses can be utilized adjusted to the objectives of the study in contrast to classical LD50 experiments.

Judged against physiological parameters like temperature or body weight, spirometry has the advantage of being more sensitive in detecting infection-induced pulmonary pathology. Alterations can be monitored for a period of time at least four times longer than that over which changes in body temperature are measureable. Additionally, the affected organ is directly monitored instead of a surrogate parameter, thus making spirometry more specific.

Disadvantages of the method are only few and of minor nature. They include size/weight limits of the mice for the inserts and the requirement for a short training period for the researcher in handling and data evaluation. However, inserts can be manufactured to meet the requirements easily, and so the measurement of other rodent species could become feasible. Mouse handling and compliance as well as data processing and evaluation can be easily learned by the researcher. 
Spirometry is a less invasive measurement than other methods making the measuring of numerous time points on the same animal possible - an advantage when utilizing strains with limited availability and expensive knockout mice. Due to the ability to utilize non-lethal infectious doses, experiments using multiple infections are feasible as performed for vaccination trials, all with the benefit of higher sensitivity, specificity and reproducibility than other methods. Although the technical procedures are somewhat more elaborate than for whole-body plethysmography, they are justified by the higher quality of data obtained. According to our data, the most meaningful parameters were the parameters 'tidal volume' and 'volume', which were altered for $72 \mathrm{~h}$ after inoculation, followed by the expiratory flows (EF50, PEF) which could be monitored up to $48 \mathrm{~h}$ after inoculation with high accuracy and reproducibility.

Further use of the method can include allergy and infection in conjunction with asthma models by easy modification of the spirometric set-up through addition of a nebulization device.
Due to the non-lethal properties of lung function measurements, the applied method could also be used in breeding programs. Mice with a desired lung phenotype can be investigated spirometrically and afterwards selected for mating.

In conclusion, the applied method of head-out spirometry holds great potential in the utilization for the investigation of lung infectious diseases in rodents as a model system and treatment platform for lung diseases in humans.

\section{Acknowledgements}

The authors wish to express their gratitude to the lab of Achim Gruber for the preparation and staining of the histological samples. We thank Sylwia Wiehlmann for excellent technical assistance and Christian Gülde for the preparation of the video file. This study was supported by Deutsche Forschungsgemeinschaft (GRK 653/3 and 653/4, SFB587, A9).

\section{References}

1 Jenkins C: COPD management. 1. Strategies for managing the burden of established COPD. Int J Tuberc Lung Dis 2008;12:586594.

- 2 Kozlowska WJ, Bush A, Wade A, Aurora P, Carr SB, Castle RA, Hoo A, Lum S, Price J, Ranganathan S, Saunders C, Stanojevic S, Stroobant J, Wallis C, Stocks J: Lung function from infancy to the preschool years after clinical diagnosis of cystic fibrosis. Am J Respir Crit Care Med 2008;178:42-49.

-3 Zapletal A, Hladíková M, Chalupová J, Svobodová T, Vávrová V: Area under the maximum expiratory flow-volume curve: a sensitive parameter in the evaluation of airway patency. Respiration 2008;75:40-47.

-4 Keochkerian D, Chlif M, Delanaud S, Gauthier R, Maingourd Y, Ahmaidi S: Breathing pattern adopted by children with cystic fibrosis with mild to moderate pulmonary impairment during exercise. Respiration 2008; $75: 170-177$

5 Newton TJ: Respiratory care of the hospitalized patient with cystic fibrosis. Respir Care 2009;54:769-776

-6 Osthoff M, Leuppi JD: Management of chronic obstructive pulmonary disease patients after hospitalization for acute exacerbation. Respiration 2010;79:255-261.

7 Niewoehner DE: Relation of chronic obstructive pulmonary disease exacerbations to $\mathrm{FEV}_{1}$ : an intricate tango. Respiration 2009;77:229-235.
8 George AM, Jones PM, Middleton PG: Cystic fibrosis infections: treatment strategies and prospects. FEMS Microbiol Lett 2009;300: 153-164.

-9 Lechtzin N, John M, Irizarry R, Merlo C, Diette GB, Boyle MP: Outcomes of adults with cystic fibrosis infected with antibioticresistant Pseudomonas aeruginosa. Respiration 2006;73:27-33.

10 van Heeckeren AM, Schluchter MD: Murine models of chronic Pseudomonas aeruginosa lung infection. Lab Anim 2002;36:291-312.

11 Glaab T, Taube C, Braun A, Mitzner W: Invasive and noninvasive methods for studying pulmonary function in mice. Respir Res 2007;8:63.

12 Martin TR, Gerard NP, Galli SJ, Drazen JM: Pulmonary responses to bronchoconstrictor agonists in the mouse. J Appl Physiol 1988; 64:2318-2323.

13 Hamelmann E, Schwarze J, Takeda K, Oshiba A, Larsen GL, Irvin CG, Gelfand EW: Noninvasive measurement of airway responsiveness in allergic mice using barometric plethysmography. Am J Respir Crit Care Med 1997;156:766-775.

14 Bates JHT, Irvin CG: Measuring lung function in mice: the phenotyping uncertainty principle. J Appl Physiol 2003;94:1297-1306.

15 Vijayaraghavan R, Schaper M, Thompson R, Stock MF, Boylstein LA, Luo JE, Alarie Y: Computer assisted recognition and quantitation of the effects of airborne chemicals acting at different areas of the respiratory tract in mice. Arch Toxicol 1994;68:490-499.

16 Rakhimova E, Munder A, Wiehlmann L, Bredenbruch F, Tümmler B: Fitness of isogenic colony morphology variants of Pseudomonas aeruginosa in murine airway infection. PLoS ONE 2008;3:e1685.

-17 Munder A, Krusch S, Tschernig T, Dorsch M, Lührmann A, van Griensven M, Tümmler B, Weiss S, Hedrich HJ: Pulmonary microbial infection in mice: comparison of different application methods and correlation of bacterial numbers and histopathology. Exp Toxicol Pathol 2002;54:127-133.

-18 Munder A, Zelmer A, Schmiedl A, Dittmar KE, Rohde M, Dorsch M, Otto K, Hedrich HJ, Tümmler B, Weiss S, Tschernig T: Murine pulmonary infection with Listeria monocytogenes: differential susceptibility of $\mathrm{BALB} / \mathrm{c}, \mathrm{C} 57 \mathrm{BL} / 6$ and $\mathrm{DBA} / 2$ mice. $\mathrm{Mi}$ crobes Infect 2005;7:600-611.

19 Sankoh AJ, Huque MF, Dubey SD: Some comments on frequently used multiple endpoint adjustments methods in clinical trials. Stat Med 1997;16:2529-2542. (SISA: www. quantitativeskills.com/sisa/calculations/ bonfer.htm)

20 Wilcoxon F: Individual comparisons by ranking methods. Biometrics 1945;1:80-83.

21 Mann HB, Whitney DR: On a test of whether one of two random variables is stochastically larger than the other. Ann Math Statist 1947;18:50-60. 